\title{
PRELIMINARY RESULTS FROM THE COSMIC BACKGROUND IMAGER
}

\author{
B.S. MASON, J.K. CARTWRIGHT, S. PADIN, T.J. PEARSON, A.C.S. READHEAD, \\ M. SHEPHERD, J. SIEVERS, P. UDOMPRASERT \\ 105-24 Caltech \\ Pasadena, CA 91125 \\ Email: bsm@astro.caltech.edu
}

\begin{abstract}
The Cosmic Background Imager (CBI) is a 13-element interferometer designed to image intrinsic anisotropies in the cosmic microwave background (CMB) on arcminute scales. A review of the capabilities of the instrument is presented, together with a discussion of observations which have been taken over the past 9 months from the Atacama desert of Chile. We present preliminary high-resolution mosaiced images of the CMB obtained from recent CBI data and discuss topics which the CBI will address in the near future.
\end{abstract}

\section{Introduction and Overview of the CBI}

Anisotropies in the Cosmic Microwave Background (CMB) contains a wealth of information about fundamental cosmological parameters 4 , as well as providing a direct link to theories of high-energy physics 1 . Inspired by the possibility of accurate determinations of classical cosmological parameters, experiments have sought and detecteted the mast prominent large-scale CMB anisotropies due to the first

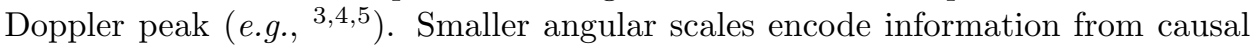
processes which are independent of the location of the Doppler peaks. The primary mission of the CBI is to measure the CMB anisotropy spectrum in this relatively unexplored regime.

The CBI is an interferometric array of 130.9 -meter diameter antennas mounted on a 6-meter steerable platform, and operating in $101-\mathrm{GHz}$ bands between 26 and $36 \mathrm{GHz}(\sim 1 \mathrm{~cm})$. Configurations available to the CBI yield synthesized beamwidths ranging from $4^{\prime}$ to $15^{\prime}$ (FWHM). Interferometry confers the significant advantage, relative to total power or beam-switched single-dish methods, of providing a direct measurement of $C_{\ell}$ on a scale determined by the baseline length. The range of baselines available to the CBI correspond to $300<\ell<3000$. By changing the array configuration of the CBI, the instrument's sensitivity can be optimized for varying ranges of $\ell$. The primary beam width $44^{\prime}$ (FWHM at $30 \mathrm{GHz}$ ) implies a resolution $\delta \ell \sim 420$ (FWHM); this can be significantly improved by mosaicked observations. Two configurations of the CBI are shown in Figure 1 .

The CBI will be capable of providing the first single-experiment measurement of the photon diffusion scale yhich is a fundamental prediction of current theories of CMB anisotropy formation 6 . Results at the low end of the range of $\ell$ available to the CBI will provide an important check on other power spectrum determinations in this range such as RING5M and BOOMERANG/MAXIMA, which are total power experiments. Two other interferometers are DASI and the VSAl, both of which operate at lower $\ell$ than the CBI. DASI is a sister experiment to the CBI with many design elements in common, and these two interferometers together cover the 

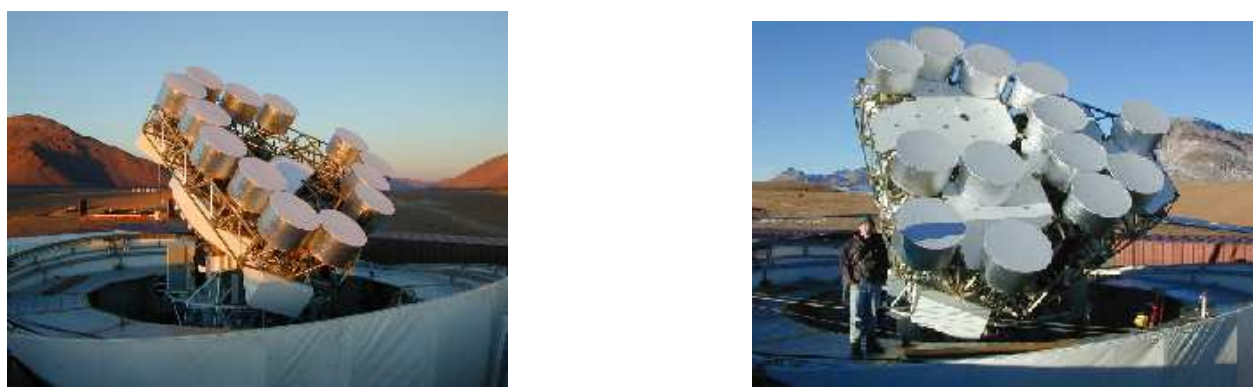

Figure 1. The CBI in its test configuration (left) providing uniform $u v$-coverage and easy access to all receivers, and in a configuration providing more short baselines for mosaic and SunyaevZel'dovich Effect observations (right).

range $100<\ell<3000$.

\section{Observing Strategy and Preliminary Results}

Construction of the CBI was begun in August 1995 in Pasadena, CA on the Caltech campus, and completed in January 1999. After a period of test observations in Pasadena, the telescope was disassembled and shipped in August 1999 to its site high in the Chilean Andes. This site, at an altitude of 5000 meters, was chosen in order that our sensitivity not be limited by atmospheric water vapor emissions. First light in Chile was achieved November 1, 1999, and routine observations have been taken from January 2000 to the present.

Our observing strategy is dictated by the fact that, on the shortest CBI baselines, the ground produces significant correlated signals. To remove these, pairs of fields are observed over identical ranges in azimuth and elevation. The difference between these data cancels ground-based signals. This differencing also controls other possible systematics due, e.g., to correlator offsets or antenna cross-talk. From January through April 2000, observations of two pairs of such fields were taken in a test configuration providing a maximally uniform distribution of baseline lengths. In all $\sim 160$ hours of integration were obtained, resulting in a robust detection of CMB anisotropy on scales from $\ell=300$ to $\ell=1500$. Results from these observations have been accepted for publication 2 .

In order to remove discrete radio sources, the dominant foreground to CMB measurements at our frequency and resolution, dedicated observations of NVSS10 selected sources are conducted at $30 \mathrm{GHz}$ with the OVRO 40-meter telescope. Sources detected by the 40-meter are subtracted directly from the CBI data.

Observations of three $5^{\circ} \times 3^{\circ}$ mosaic fields are in progress. When complete, these results will improve our resolution in $\ell$ by a factor of $3-4$. Figure 2 shows a difference image of the CMB from preliminary observations of one of our three mosaic fields before and after the removal of discrete source contamination. 

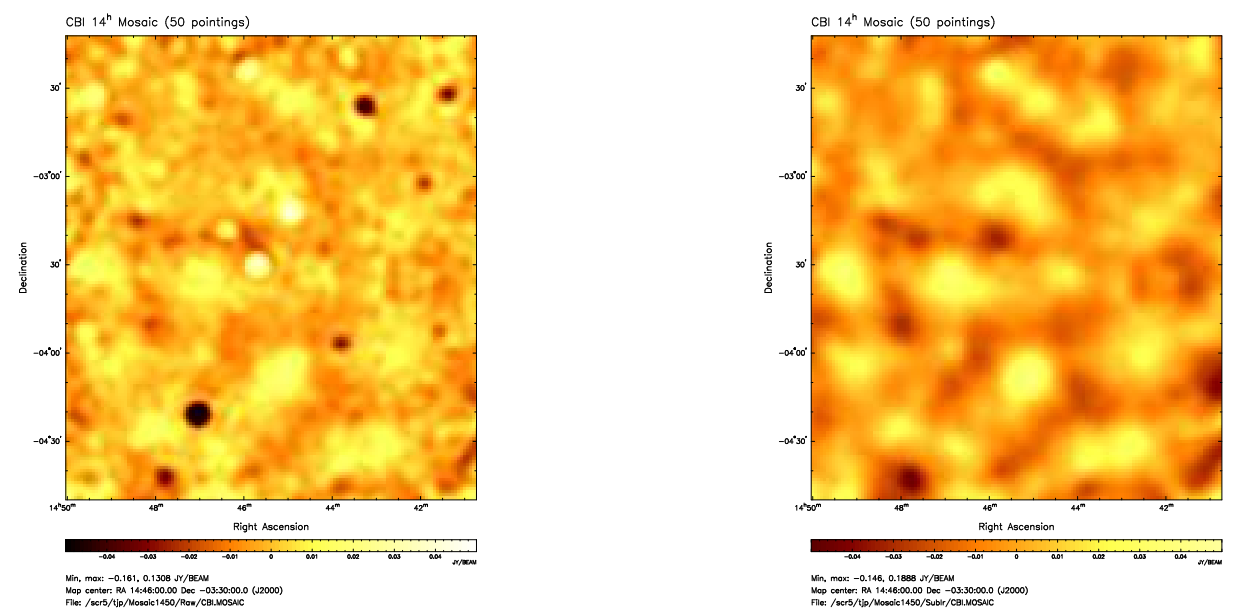

Figure 2. Images from observations of one mosaic field. On the left, the raw mosaic data are shown, including contaminating discrete sources. The image on the right has had discrete sources removed via independent observations with the OVRO $40-\mathrm{m}$, and the long baseline data are downweighted to emphasize the large-scale structure in the field. Most of this is due to intrinsic CMB fluctuations.

\section{Other Science with the CBI}

The CBI has one cross-polarized antenna. This will enable stringent limits to be placed on the CMB polarization in the vicinity of the first Doppler peak in the polarization power spectrum. Polarization data have been acquired on the CBI fields from January 2000 through the present.

The range of angular scales available to the CBI are also well-suited to measuring the Sunyaev-Zeldovich Effect (SZE) in nearby galaxy clusters. A campaign to determine $\mathrm{H}_{0}$ from observations of the SZE in a sample of $20 z<0.1$ clusters is underway with the CBI. This campaign has the feature, unique amono surrent SZE programs, of selecting targets from an orientation-unbiased sample 11, 12 . The large sample size is important for reducing the effects of intrinsic CMB anisotropy, and for understanding possible X-ray modelling systematics associated with clusters at a range of dynamical states.

\section{Acknowledgments}

The CBI is a project of the California Institute of Technology, in collaboration with the Universidad de Chile. The construction of the CBI has been made possible by the generous support of the California Institute of Technology, Ronald and Maxine Linde, Cecil and Sally Drinkward, and grants from the National Science Foundation (awards AST-9413935 and AST-9802989). 


\section{References}

1. White, M., Scott, D. \& Silk, J., Ann. Rev. A \& A 32, 319 (1994)

2. Kamionkowski, M. \& Kosowski, A., Ann. Rev. Nucl. Part. Sci. 49, 77 (1999)

3. Miller, A.D. et al., ApJ 524, L1 (1999)

4. Leitch, E.M. et al., ApJ 532, 37 (2000)

5. de Bernardis, P. et al., Nature 404, 955 (2000)

6. Silk, J., ApJ 151, 459 (1968)

7. Halverson, N.W., Carlstrom, J.E., Dragovan, M., Holzapfel, W.L., and Kovac, J., SPIE 3357, 416 (1998)

8. Grainge, K. et al., this volume.

9. Padin, S. et al., ApJL in press (astro-ph/0012212)

10. Condon, J. et al., AJ 116, 1693 (1998)

11. Udomprasert, P.S., Mason, B.S., \& Readhead, A.C.S., to appear in Constructing the Universe with Clusters of Galaxies, ed. F. Duret and D. Gerbel, "The Sunyaev-Zel'dovich Effect with the Cosmic Background Imager."

12. Mason, B.S. \& Myers, S.T., ApJ 540, 614 (2000) 